\title{
Where do you Find Interesting Biosurveillance Publications?
}

\author{
Katie J. Suda*1, Tera Reynolds ${ }^{2}$, Judy Akkina ${ }^{3}$, Sylvia Halasz ${ }^{4}$ and Howard Burkom ${ }^{5}$ \\ 1VA/UIC, Chicago, IL, USA; ${ }^{2}$ ISDS, Boston, MA, USA; ${ }^{3}$ USDA, Fort Collins, CO, USA; ${ }^{4} \mathrm{yp}$, Glendale, CA, USA; $5 \mathrm{JHU} / \mathrm{APL}, \mathrm{Laurel}, \mathrm{MD}$, \\ USA
}

\section{Objective}

To identify the disciplines and journal titles of surveillance-related publications from a wide range of indexed repositories and to draw attention to the publication repository created by the ISDS Research Committee.

\section{Introduction}

The ISDS Research Committee (RC) is an interdisciplinary group of researchers interested in a wide range of topics related to disease surveillance. The RC hosts a literature review process that results in a permanent repository ${ }^{1}$ of relevant journal articles; some of which are presented in bi-monthly calls/webinars that provide a forum for discussion and author engagement. ${ }^{2}$ The webinars have led to workgroups and society-wide events, boosted interest in ISDS, the annual conference, and fostered networking among members and guests.

Since 2007, the RC has identified and classified published articles using an automated search method with the aim of progressing ISDS's mission of advancing the science and practice of disease surveillance by fostering collaboration and increasing awareness of current advances in the field of surveillance. In 2012 the RC refined the method of automated literature retrieval resulting in increases in relevant articles identified. The RC literature review efforts have provided an opportunity for interdisciplinary collaboration and have resulted in a repository of 1920 articles from March 2012-August $2014(2012=37.4 \%$ of articles in the repository, $2013=35.1 \%$, $2014=27.5 \%$ ).

\section{Methods}

A search query was developed in Scopus, ${ }^{3}$ consisting of over 100 terms suggested by members. We found that the Scopus search is the most comprehensive and improved the cross-disciplinary scope. Scopus results allowed filtering of 50-100 titles and abstracts in fewer than 30 minutes each week for the identification of articles of interest to ISDS members.

Journal titles were categorized to identify the primary discipline covered by the journal; categories include health sciences, mathematics/physics/statistics ("math"), computer science, animal/ plant, geography/environment, engineering, business/operations/ organizational research ("business"), and other science (e.g., biology). Conference abstracts were categorized separately. Journals with a primary focus of surveillance were also indicated in the dataset.

\section{Results}

The majority (66.9\%) of articles in the repository were published by journals with a focus in health science, followed by math (11.0\%), geography/environment $(6.5 \%)$, computer science $(5.7 \%)$, other science $(4.9 \%)$, and animal/plant $(3.8 \%)$. Although few articles were published in engineering (1.6\%) and business $(0.3 \%)$, these are emerging areas in surveillance and should continue to be monitored for work impacting the field. Only $0.03 \%(n=14)$ articles in the repository could not be categorized. Journals with the highest frequency of surveillance articles included PLOS ONE (21.4\% of articles in the repository), Influenza and Other Respiratory Viruses (6.1\%), Vaccine (3.3\%), and Preventive Veterinary Medicine (3.1\%). Journals with a primary focus of surveillance included EuroSurveillance, MMWR Surveillance Summaries, and Surveillance and Society; publishing $1.7 \%(\mathrm{n}=33)$ of articles in the repository.

\section{Conclusions}

Although the majority of articles are published in health science journals, one-third of articles relevant to surveillance are published in other disciplines not indexed by traditional secondary databases (e.g., Medline). Researchers and public health professionals working in the area of surveillance should use the article repository to identify articles relevant to their work and avoid missing studies outside of the health sciences.

\section{Keywords}

biosurveillance; publications; literature search; literature query

\section{Acknowledgments}

The ISDS RC would like to thank many past contributors and guest authors to the literature review process.

\section{References}

1. Zotero. http://www.webcitation.org/6AZia0yEd.

2. ISDS Literature Review Summaries Archive. ISDS Wiki. http://www. webcitation.org/6AZkiT9xQ.

3. ISDS Literature Review Search String (Scopus). http://www. webcitation.org/6AZiWfC6C.

*Katie J. Suda

E-mail: katiesuda@gmail.com 